\title{
Controlling electrostatic co-assembly using ion-containing copolymers : from surfactants to nanoparticles
}

\author{
J.-F. Berret \\ Matière et Systèmes Complexes, UMR 7057 CNRS Université Denis Diderot Paris-VII, Bâtiment \\ Condorcet, 10 rue Alice Domon et Léonie Duquet, 75205 Paris (France)
}

\begin{abstract}
In this review, we address the issue of the electrostatic complexation between chargedneutral diblock copolymers and oppositely charged nanocolloids. We show that nanocolloids such as surfactant micelles and iron oxide magnetic nanoparticles share similar properties when mixed with charged-neutral diblocks. Above a critical charge ratio, core-shell hierarchical structures form spontaneously under direct mixing conditions. The core-shell structures are identified by a combination of small-angle scattering techniques and transmission electron microscopy. The formation of multi-level objects is driven by the electrostatic attraction between opposite charges and by the release of the condensed counterions. Alternative mixing processes inspired from molecular biology are also described. The protocols applied here consist in screening the electrostatic interactions of the mixed dispersions, and then removing the salt progressively as example by dialysis. With these techniques, the oppositely charged species are intimately mixed before they can interact, and their association is monitored by the desalting kinetics. As a result, sphere- and rod-like aggregates with remarkable superparamagnetic and stability properties are obtained. These findings are discussed in the light of a new paradigm which deals with the possibility to use inorganic nanoparticles as building blocks for the design and fabrication of supracolloidal assemblies with enhanced functionalities.
\end{abstract}

outline

I - Introduction

II - Electrostatic Complexation between oppositely charged surfactants and copolymers

II.1 - Homopolyelectrolytes and surfactants

II. 2 - Evidence of critical mixing ratios

II.3 - Hierarchical structures revealed by neutron scattering

III - Generalization of the complexation protocols to inorganic nanoparticles

III.1 - Existing clustering strategies for inorganic nanoparticles

III.2 - Iron oxide nanoparticles as a model system

III.3 - Iron oxide supracolloidal assembly

IV - Desalting transition : towards the fabrication of controlled size clusters and rods

IV.1 - Alternative protocols for mixing species with opposite charges

IV.2 - Evidence of a desalting transition as a function of ionic strength

IV.3 - Magnetic clusters and rods

$\mathrm{V}-$ Conclusion

Submitted to Advanced Colloid and Polymer Science

*Corresponding author jean-francois.berret@univ-paris-diderot.fr

\section{1 - Introduction}

The emergence of novel materials and processing at the nanoscale has set the conditions for the fabrication of a wide range of nano-objects and multilevel nanostructured networks [1-3]. In particular, the co-assembly of polymers and nanoparticles is opening pathways for engineering versatile hybrid structures combining the advantageous properties of both the organic and inorganic worlds. Interest stems from the combination of complementary attributes, such as a size in the nanometer range and 
unique physical features including high reactivity or magnetic and optical properties. With respect to assembly mechanisms, electrostatic complexation between oppositely charged macromolecular objects has gained special interest over the last years [4]. Electrostatic complexation designates the process by which co-assembly is driven by the pairing of electric charges located e.g. at the surfaces of particles or along the backbones of polymers.

This research was motivated by the fact that electrostatics is at the origin of many non-specific associations relevant in biological systems, such as protein complexes or chromatin. The chromatin is described as the lowest hierarchical level of chromosomes in eucaryotic cells. The basic repeating unit of chromatin fibers, the nucleosomes can be reconstituted in vitro from its pure components, mainly histones and DNA by various techniques [5]. This reconstitution process can be seen as the electrostatic complexation between an excess of polyanions, here 146 base pairs of DNA per nucleosome and a limited set of polycations, i.e. an octamer of cationic histones [6, 7].

A second prominent application of electrostatic complexation concerns polyelectrolyte multilayers [8, 9]. The layer-by-layer deposition of oppositely charged polyelectrolytes is a remarkable illustration of a supramolecular assembly at interfaces. Using this interfacial complexation approach, researchers have generated multicomponent or multilayer assemblies composed of different charged macromolecules [10, 11] and/or inorganic nano-objects such as particles, tubes, proteins $[12,13]$ resulting in thin films with high capacitance property [14] or coatings with antireflection and self-cleaning properties $[15,16]$.

A third class of systems where electrostatic complexation is at stake is that of charged-neutral block copolymers, also called double hydrophylic block copolymers. Unlike amphiphilic copolymers, charged-neutral blocks are soluble in aqueous media, but co-assemble in the presence of oppositely charged species. These copolymers were popularized in the mid 1990's by the group of Kataoka who was the first to suggest the formation of polyion complex micelles from a pair of oppositely charged block polypeptides with poly(ethylene glycol) segments [17-20]. Since this first attempt, it was recognized that the attractive interactions between copolymers and oppositely charged species generate new type of colloids $[4,21]$. These colloids form spontaneously under direct mixing and exhibit a coreshell microstructure. The formation of mixed aggregates is generally described as the result of a nucleation and growth mechanism of a microphase made from the oppositely charged constituents [22]. Owing to the hindrance barrier represented by the shell, the growth is arrested at a size which is fixed by the dimension of the polymer. The specimens examined so far comprise synthetic [23-27] and biological $[18,28]$ macromolecules, multivalent counterions [29, 30], surfactant micelles [31-33, 22, 34-39]. Electrostatic complexation is also utilized in numerous applications such as formulation of personal care products, water treatment and filtration, coating, drug delivery, bioelectronics and nanocomposite technologies $[4,21]$.

Concerning the complexation mechanism, it is now accepted that the driving forces for association are both enthalpic and entropic in origin [40-43, 9]. This is true for homopolyelectrolytes as well as for the aforementioned charged-neutral block polymers. The enthalpic part in the free energy of association is linked to the pairing of the opposite charges. The binding enthalpy depends strongly on the chemical constituents that are paired in the complexation [9]. The entropic contribution to the free energy arises from the release of the counterions which are condensed on the surface of the colloid or along the backbone of the polymer [44], as well as from the loss of translational and rotational degrees of freedom of macromolecules in their bound state [45]. The balance between the enthalpic and entropic contributions gives rise to wide variety of complexation behaviors. The association can be strong, and then results in the formation of coacervates and macroscopic phase separations [46-48, 37]. In some systems, the complexation with ion-containing chains is weaker and the electrostatic complexes remain soluble $[49,50,43,51-53]$. For electrostatic complexes however, it is essential to know their microstructures and stoichiometry since these properties will ultimately determine their range of applications [21].

In the present review, we focus on electrostatic complexation between charged-neutral diblock copolymers and oppositely charged nanocolloids such as surfactant micelles (section II) and magnetic 
nanoparticles (Section III). For both types of nanocolloids, core-shell hierarchical aggregates of sizes comprised between 20 and $500 \mathrm{~nm}$ were found under direct mixing conditions. In Section IV, we discuss alternative protocols for bringing oppositely charged species together. The association between charged-neutral copolymers and nanocolloids dispersed in brine solutions can be monitored by controlling the desalting kinetics. As a result, sphere- and rod-like aggregates with remarkable superparamagnetic and stability properties were obtained.

\section{II - Electrostatic Complexation between oppositely charged surfactants and copolymers}

\section{II.1 - homopolyelectrolytes and surfactants}

When homopolyelectrolytes are mixed to oppositely charged surfactants in aqueous solutions, two phenomena are successively observed. First, at very low polymer concentration the surfactants and polyelectrolytes co-assemble into aggregates similar to surfactant micelles. This phenomenon occurs at the critical aggregation concentration (cac) which is usually much lower than the regular critical micellar concentration $(\mathrm{cmc})[54,55,50]$. As the surfactant concentration is further increased, a phase separation is observed. The solution becomes turbid at the mixing of the two components, and after centrifugation it displays two separated phases. The bottom phase appears as a concentrated liquid phase or as a solid precipitate whereas the supernatant remains fluid and transparent. The conditions for the phase separation are that the ratio $\mathrm{Z}$ between the positive and negative charges is above a critical value $Z_{C}$, and that the salt content remains low [55]. Note that the adsorption of such electrostatic coacervate phases at liquid-solid interfaces were also reported. They revealed synergistic co-adsorption features and non equilibrium behaviors that can be exploited in the context of coating and anti-biofouling applications [56-58].

In recent years, increasing attention was placed on the structure of the concentrated solid phase because it is anticipated that the description of the precipitates is a prerequisite for the understanding of the complexation mechanisms. Using small-angle x-ray scattering techniques, it was shown that the solid precipitates exhibited liquid crystalline order reminiscent from the structures found in surfactant concentrated phases $[46,59,47,60-66,48,37]$. Cubic, hexagonal and lamellar phases were identified, depending on if the surfactant self-assembled into spheres, cylinders and bilayers, respectively. In these crystalline mesophases, the polyelectrolyte chains are assumed to be adsorbed at the surface of the aggregates and to link them $[47,62,48]$.

When the former ion-containing chain is now one part of a diblock copolymer, the other block being a neutral and water soluble chain, the phenomena described above are modified. Aqueous solutions of asymmetric diblocks with oppositely charged surfactants do not exhibit a phase separation. Instead, there is the formation of finite size colloids made from both surfactant and copolymers. Many different names were given to these aggregates. Colloids made with surfactants were dubbed block ionomer complexes [31], colloidal or electrostatic complexes [22, 34, 37, 39]. Co-assembled colloids made from oppositely charged block polypeptides or from proteins were called polyion complex micelles by Harada and coworkers $[17,18]$. Those made solely from polymers were termed (inter)polyelectrolyte complexes [67] or complex coacervate core micelles [68,4]. With surfactants, Kabanov and coworkers suggested the formation of a core-shell supramolecular assembly where the shell is made from the neutral blocks $[31,32,36]$. Using freeze fracture, these authors found that poly(ethylene oxide)- $b$ poly(sodium methacrylate) diblock copolymers and single tail surfactants arrange spontaneously into vesicles composed on closed bilayers from polymer bound surfactant arrays [69, 36].

\section{II.2 - Evidence of a Critical mixing ratio}

In this part, we illustrate our approach of controlled co-assembly by considering the ternary system composed of an anionic-neutral block copolymers, a cationic surfactant and water. The polymer was a 
poly(acrylic acid)- $b$-poly(acrylamide) with molecular weights $6500 \mathrm{~g} \mathrm{~mol}^{-1}$ and $37000 \mathrm{~g} \mathrm{~mol}^{-1}$ for each block respectively, abbreviated $\mathrm{PANa}_{6.5 \mathrm{~K}}-b-\mathrm{PAM}_{37 \mathrm{~K}}$ in the following [38]. The polymers were synthesized by Rhodia using the Madix technology for the formulation of personal care products [70]. The cationic surfactant, dodecyltrimethylammonium bromide (DTAB) is characterized by a C12 aliphatic chain and exhibits an hexagonal mesophase at high concentrations [71]. Its critical micellar concentration (cmc) in $\mathrm{H}_{2} \mathrm{O}$ is 0.46 wt. \% $(15 \mathrm{mM})$ [72].

The mixing of oppositely charged species was controlled by pouring rapidly a surfactant solution into a stock polymer solution at the desired charge ratio. This protocol, also called direct mixing will be compared to other formulation techniques in Section IV. The relative amount of each component was monitored by the charge ratio $\mathrm{Z}, \mathrm{Z}=[\mathrm{S}] / \mathrm{n}_{\mathrm{PE}}[\mathrm{P}]$ where $[\mathrm{S}]$ and $[\mathrm{P}]$ are the molar surfactant and polymer concentrations and $\mathrm{n}_{\mathrm{PE}}(=90)$ is the degree of polymerization of the polyelectrolyte block. The procedure consisting in mixing stock solutions at the same concentration was preferred because it allowed to explore a broad range of charge ratios and keep the concentration in the dilute regime, typically $\mathrm{c}<1 \mathrm{wt} . \%[35,37,38]$. The procedure also revealed the existence of a critical Z-value, noted $\mathrm{Z}_{\mathrm{C}}$ in the complex formation.

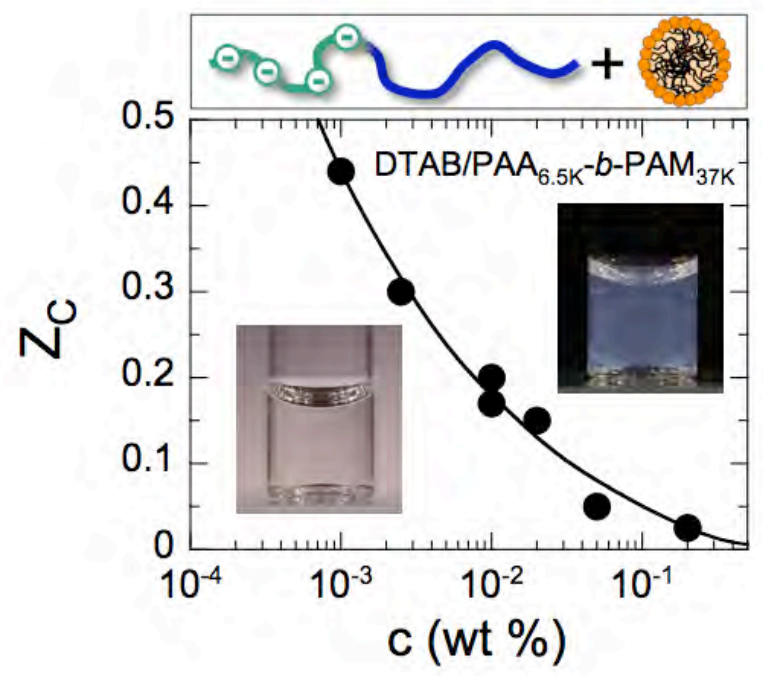

Figure 1 : Phase behavior of dispersions containing cationic surfactants (dodecyltrimethyl ammonium bromide, DTAB) and the anionic-neutral block copolymers $\left(P A N a_{6.5 K}-b-P A M_{37 K}\right)$ at $p H 8$ and $T=25{ }^{\circ} \mathrm{C}$ [22]. Above a critical mixing ratio $Z_{C}$ (close symbols), the dispersions were strongly light scattering, as illustrated by the upper right inset. The critical charge ratio $Z_{C}$ as a function of the total concentration $c$ was determined from static light scattering. The continuous line resulted from best fit calculations using $Z_{C}(c)=0.01 \times c^{-0.6}$. The panel above the figure illustrates the system considered in Section II for Figs. 1 to 5 .

Fig. 1 shows the critical charge ratio $Z_{C}$ as a function of the total concentration c. $Z_{C}$-values were derived from static light scattering experiments. They corresponded to the charge ratios where the scattering intensity exhibited a steep jump, increasing by 2 orders of magnitude $[22,37]$. The results were obtained for $\mathrm{c}=0.1 \mathrm{wt} . \%$ to $20 \mathrm{wt} . \%$ and showed that $Z_{\mathrm{C}}$ decreases with increasing $\mathrm{c}$ as a power law : $Z_{C}(c)=0.01 \times c^{-0.6}$ (continuous line in Fig. 1). These values coincided with the onset of phase separation found with the homopolyeletrolyte $\mathrm{PAA}_{5 \mathrm{~K}}$ [22]. In parallel, dynamic light scattering was performed as a function of $\mathrm{Z}$ and the hydrodynamic diameters were derived. Fig. 2 displays the evolution of the hydrodynamic diameter for $\mathrm{c}=0.1 \%$ and $1 \mathrm{wt} . \%$ as determined by dynamic light scattering using a Brookhaven spectrometer (BI-9000AT) operating at scattering angle $\theta=90^{\circ}$ and incident wave-length $\lambda=488 \mathrm{~nm}$. In this figure, three scattering regimes were distinguished. At low $\mathrm{Z}$, $\mathrm{D}_{\mathrm{H}}$ is $10 \mathrm{~nm}$, a value consistent with the hydrodynamic diameter of a single diblock molecule [22]. Slightly above $\mathrm{Z}_{\mathrm{C}}(=0.20)$, the autocorrelation function revealed the coexistence of two diffusive modes indicated by the dashed line : a fast mode associated to the diffusion of single diblock molecules, and a 
slow mode related to the diffusion of colloids of larger sizes. Between $Z_{C}$ and $1, D_{H}$ passed through a maximum and stabilized at $62 \pm 8 \mathrm{~nm}$ over a broad range of charge ratios. The colloids evidenced above $\mathrm{Z}_{\mathrm{C}}$ are those of interest in the present review.

To gain a deeper insight of their microstructure, cryo-transmission electron microscopy conducted out on a sample prepared again at isoelectric charge (Fig. 3) [35]. It revealed a existence of faintly contrasted spherical objects of average diameter $26 \mathrm{~nm}$ and polydispersity 0.16 (inset of Fig. 3). The value of $26 \mathrm{~nm}$ lied well below the actual hydrodynamic diameter measured by light scattering namely, $\mathrm{D}_{\mathrm{H}}=62 \mathrm{~nm}$. This suggested that the colloids observed by cryo-TEM were actually surrounded by a polymer shell made from the neutral blocks of poly(acrylamide). Due to the low electronic contrast of poly(acrylamide) in water, the organic shell was not detected [35]. It is worth mentioning that the shell thickness $h$ derived here $(h=17 \mathrm{~nm})$ was comparable to the hydrodynamic diameter of a single diblock $\left(\mathrm{D}_{\mathrm{H}}=11 \mathrm{~nm}\right)$.
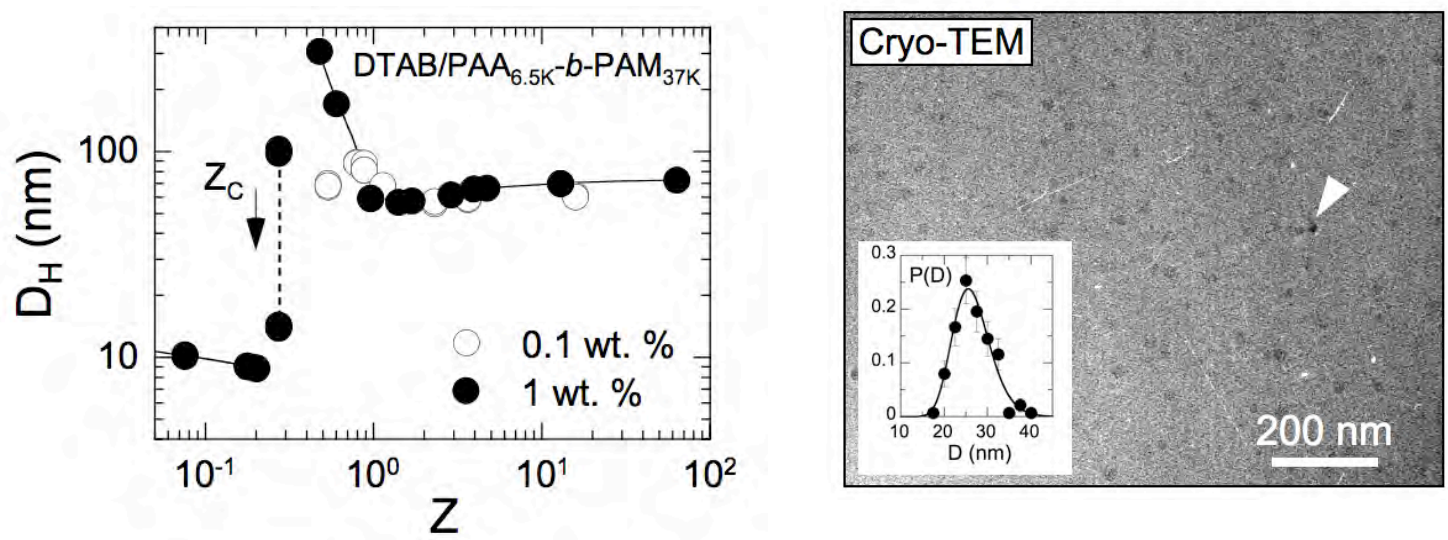

Figure 2 (left) : Evolution of the hydrodynamic diameter $D_{H}$ as function of the charge ratio $Z$ for $D T A B / P A N a_{6.5 K^{-}} b-P A M_{37 K}$ mixed dispersions at $c=0.1 \mathrm{wt} . \%$ (open symbols) and at $1 \mathrm{wt} . \%$ (closed symbols) [22]. For $Z>1, D_{H}$ reached a constant value around $62 \mathrm{~nm}$. In this experiment, each data point represents a dispersion prepared by direct mixing (see text).

Figure 3 (right) : Cryo-transmission electron microscopy image of DTAB/PANa $a_{6.5 K}-b-P A M_{37 K}$ electrostatic complexes prepared in $\mathrm{D}_{2} \mathrm{O}$ at isoelectric charge $(Z=1)$ and concentration $c=0.5 \mathrm{wt} \%$ [35]. Faintly contrasted objects (arrow) of average diameter $26 \mathrm{~nm}$ and polydispersity 0.16 are visible. Their size distribution is provided in the inset. As a result of an analysis based on light, neutron and $x$ ray scattering experiments, the objects seen by Cryo-TEM were ascribed to the cores of the electrostatic complexes illustrated in Fig. 5.

\section{II.3 - Hierarchical microstructure revealed by neutron scattering}

Because it allowed the quantitative determination of microstructures in the range $1-100 \mathrm{~nm}$, smallangle neutron scattering (SANS) was extensively used during the last decades [73]. Small-angle neutron scattering was performed at the Institute Laue-Langevin (Grenoble, France) on the beam lines D11 and D22 on samples prepared with $\mathrm{D}_{2} \mathrm{O}$ for contrast reasons. Fig. 4 shows the neutron scattering intensity obtained for a DTAB/PANa $6.5 \mathrm{~K}-b-\mathrm{PAM}_{37 \mathrm{~K}}$ solution in $\mathrm{D}_{2} \mathrm{O}$ at isoelectric charge (open symbols). Also included are the Rayleigh ratio data measured by static light scattering (closed symbols). Representative for this class of materials, the scattering intensity was characterized by three primary features :

1. A strong forward scattering as $\mathrm{q} \rightarrow 0$,

2. An oscillation at intermediate wave-vector and

3. A structure peak indicated by an arrow around $\mathrm{q}^{*}=0.165 \AA^{-1}$.

As shown using a Porod representation [22, 34], the part of the scattering corresponding to Points 1 and $2\left(\mathrm{q}<0.05 \AA^{-1}\right)$ was identified unambiguously as arising from spherical colloids of average size $22 \mathrm{~nm}$ and polydispersity 0.16 . According to this interpretation, the oscillation around $q=0.04 \AA^{-1}$ marked the 
location of the first minimum of the sphere form factor. The agreement between the cryo-TEM and SANS data for the determination of the typical colloidal sizes ( $26 \mathrm{~nm}$ versus $22 \mathrm{~nm}$ ) was here excellent.

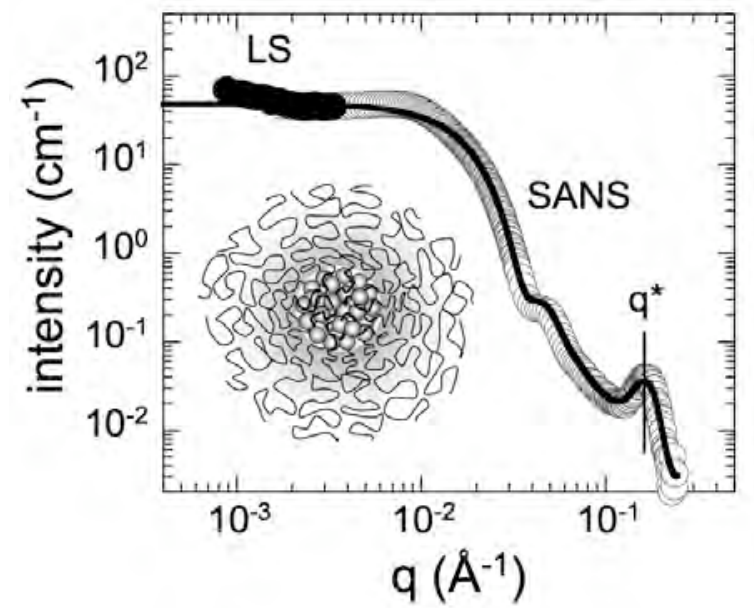

Figure 4 : Neutron scattering intensity obtained for a DTAB/PANa $a_{6.5 K}-b-P A M_{37 K}$ solution in $D_{2} O$ at isoelectric charge (open symbols). Static light scattering data are also included (closed symbols). The structure peak at $q^{*}=0.165 A^{-1}$ arises from a micro phase-separated state of strongly interacting micelles located in the core of the colloids. The continuous line results from Monte Carlo simulations using a model of colloid provided in the inset. Due to the low scattering contrast of the poly(acrylamide) in $\mathrm{D}_{2} \mathrm{O}$, the shell contribution was omitted in the simulations. Adjusting parameters are the number of micelles in the core (108) and the volume fraction (0.50) [38].

Concerning the structure peak at large wave-vectors (Point 3), several controls were performed to ascertain its origin. It was first shown to be independent on the concentration and on the charge ratio Z, and to be correlated with the strong forward scattering. Complementary experiments using deuterated surfactants or surfactants with longer aliphatic chains allowed us to draw the following conclusion : the structure peak at $\mathrm{q}^{*}$ arose from a concentrated phase of strongly interacting surfactant micelles located in the core of the aggregates. The fact that $2 \pi / \mathrm{q}^{*} \sim 4 \mathrm{~nm}$, that is the diameter of a single DTAB micelle, reinforced the assumption of closely packed micelles. By analogy with the surfactant-polyelectrolyte coacervate phases, it was suggested that the micelles were linked together by the anionic blocks, the neutral segments being outside the core and dangling in the solvent. A representation of electrostatic complexes made from surfactants and copolymers is provided in the inset of Fig. 4 [22, 34]. This construct was said hierarchical because it involves two different length scales, the size of the single surfactant micelles $(\sim 4 \mathrm{~nm})$ and that of the overall aggregate. This model was later on supported by Monte Carlo simulations [74, 35, 38, 75]. Simulations were employed to compute the scattered intensity arising from clusters of micelles. Due to the low scattering contrast of the poly(acrylamide) in $\mathrm{D}_{2} \mathrm{O}$ [38], the contribution coming from the shell was neglected in the fitting. The adjusting parameters were the distribution of aggregation numbers, i.e. the number of spheres enclosed into the core and the volume fraction inside the core. The continuous line in Fig. 4 was obtained using an average of 108 micelles by core and a volume fraction of 0.50 . The model accounted simultaneously for the three features mentioned previously (Points 1-3).

In a later study, copolymers of different molecular weights and chemistry were investigated [37]. It was found that the core sizes of the complexes depended essentially on the structural charges borne by the charged block. In terms of stability, the optimal conditions were reached when the degree of polymerization of the neutral block was 2 - 5 times that of the charged block. Since our first SANS survey on the internal structure of surfactant-based electrostatic complexes, numerous colloidal systems with structure peaks at high wave-vectors were reported in the literature, and supported our view. This included surfactants [38, 76, 39, 77], proteins [78] or particles [75]. In conclusion, we show that oppositely charged surfactant and double hydrophilic block copolymers spontaneously co-assembled in 
the form of spherical hierarchical colloids, following a mechanism illustrated in Fig. 5. The question arises whether the above strategies could be generalized to other nanocolloids such as inorganic nanoparticles?

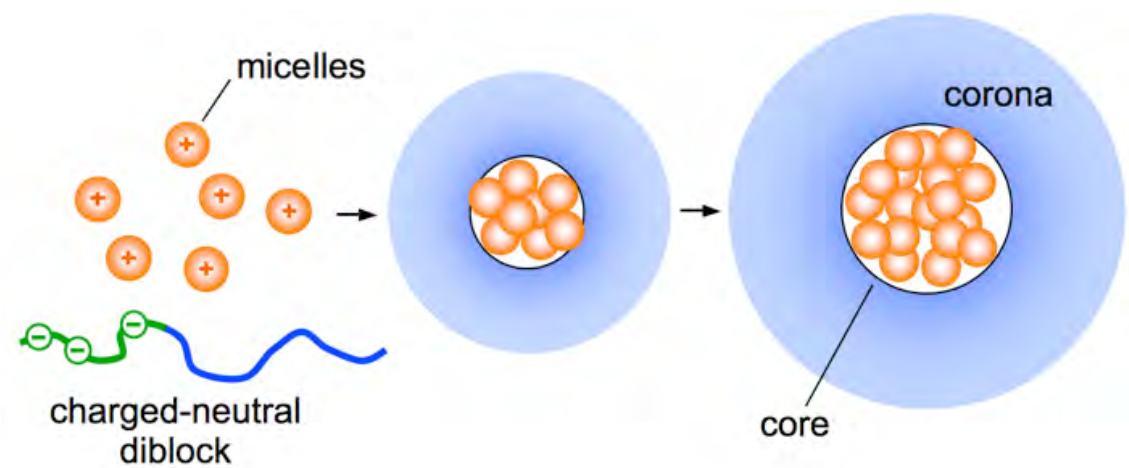

Figure 5 : Schematic representation of the complex formation using oppositely charged surfactants and block copolymers.
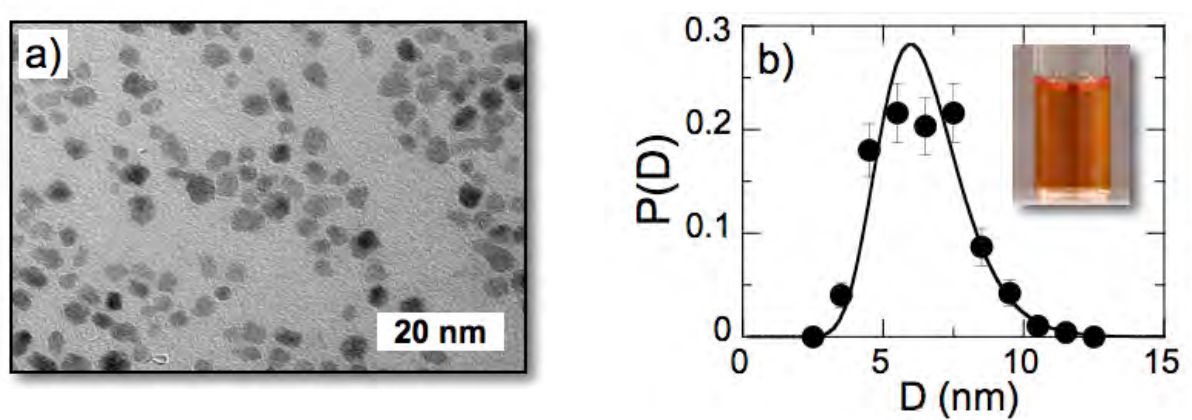

Figure 6 : Upper panel : transmission electron microscopy (TEM) images of iron oxide nanoparticles investigated in this work, magnification $\times 120000$. Lower panel : size distributions was derived from TEM images and fitted using a log-normal function, with median diameter $6.3 \mathrm{~nm}$ and polydispersity 0.23 [100]. The polydispersity was defined as the ratio between the standard deviation and the average. The inset shows a photograph of a vial containing a dilute iron oxide dispersion, $c=0.2 \mathrm{wt} . \%$.

\section{III - Generalization of the complexation protocols to inorganic nanoparticles}

\section{III.1 - Existing Clustering strategies for inorganic nanoparticles}

Inorganic nanoparticles made from noble metals, from oxides or from semiconductors are characterized by sizes comprised between 1 and $100 \mathrm{~nm}$ [79-81, 3]. In applications however, particulates with larger sizes may be required but yet remain difficult to generate by soft chemistry. Several issues become critical when the particle size increases, including colloidal stability, sedimentation and loss of physical properties. In order to circumvent these limitations, co-assembly strategies involving sub-10 nm nanoparticles were elaborated [82-92]. Nanoparticles were arranged into clusters of different dimensions and morphologies, with aggregation numbers ranging from few units to several thousands [82, 84, 87, 93, 89-92]. Because many nanomaterials are available in aqueous media, and because most of them display electric charges at their surfaces, the challenge was to generalize the approach developed for surfactants to inorganic particles. Concerning our work, sub-10 nm particles such as cerium oxide $\left(\mathrm{CeO}_{2}\right.$, nanoceria) [94-98], iron oxide $\left(\gamma-\mathrm{Fe}_{2} \mathrm{O}_{3}\right.$, maghemite) [99-102, 95, 103, 104], europium-doped yttrium vanadate $\left(\mathrm{Eu}: \mathrm{YVO}_{4}\right)[90]$ and yttrium hydroxyacetate $\left(\mathrm{Y}(\mathrm{OH})_{1.7}\left(\mathrm{CH}_{3} \mathrm{COO}\right)_{1.3}\right)$ 
$[87,105]$ were studied. As an illustration, we focus here on the model system made from magnetic nanoparticles $\left(\gamma-\mathrm{Fe}_{2} \mathrm{O}_{3}\right)$.

\section{III.2 - Magnetic Nanoparticles as a model system}

Iron oxide nanoparticles (bulk mass density $\rho=5100 \mathrm{~kg} \mathrm{~m}^{-3}$ ) were synthesized according to the Massart technique [106, 107] by alkaline co-precipitation of iron(II) and iron(III) salts, oxidation of the magnetite $\left(\mathrm{Fe}_{3} \mathrm{O}_{4}\right)$ into maghemite $\left(\gamma-\mathrm{Fe}_{2} \mathrm{O}_{3}\right)$ nanoparticles, and by size-sorting by subsequent phase separations $[108,107]$. At the end of the synthesis, the particles were dispersed in water at a weight concentration $\mathrm{c} \sim 10 \mathrm{wt} . \%$ and $\mathrm{pH} 1.8$. At this $\mathrm{pH}$, the particles were positively charged. The resulting electrostatic repulsion insured the colloidal stability of the dispersions over period longer than years. The magnetic fluids were characterized by electron microdiffraction, vibrating sample magnetometry, magnetic sedimentation and light scattering. For the dispersions investigated here, the size distribution as determined from TEM measurement (Fig. 6a) could be represented by a log-normal function, with median diameter $\mathrm{D}_{0}^{\mathrm{NP}}=6.3 \mathrm{~nm}$ and polydispersity $\mathrm{s}^{\mathrm{NP}}=0.23$ (Fig. 6b) [100]. The particles were further functionalized with citrate ligands, a procedure which allowed to reverse the surface charges from cationic at low $\mathrm{pH}$ to anionic at high $\mathrm{pH}$ through the ionization of the carboxyl groups. For the citrate coated particles $\left(\mathrm{Cit}-\gamma-\mathrm{Fe}_{2} \mathrm{O}_{3}\right)$, the structural charge density was ascertained at $-2 e \mathrm{~nm}^{-2}$ by conductivity $[109,110]$ and light scattering measurements [90]. Cit- $\gamma-\mathrm{Fe}_{2} \mathrm{O}_{3}$ nanoparticles had thus sizes and surface charge densities that are larger than those of surfactant micelles ( 6.3 versus $4 \mathrm{~nm}$ for the diameter, $-2 e$ versus $+1 e \mathrm{~nm}^{-2}$ for the density). Taking these figures into account, the inorganic particles had 5 times more structural charges at their surfaces as compared to surfactant aggregates (250 e versus $+53 e$ ) [90]. The sign of the electrostatic charges between the cationic micelles and the citrate-coated nanoparticles was also different, and cationic-neutral copolymers were used instead.
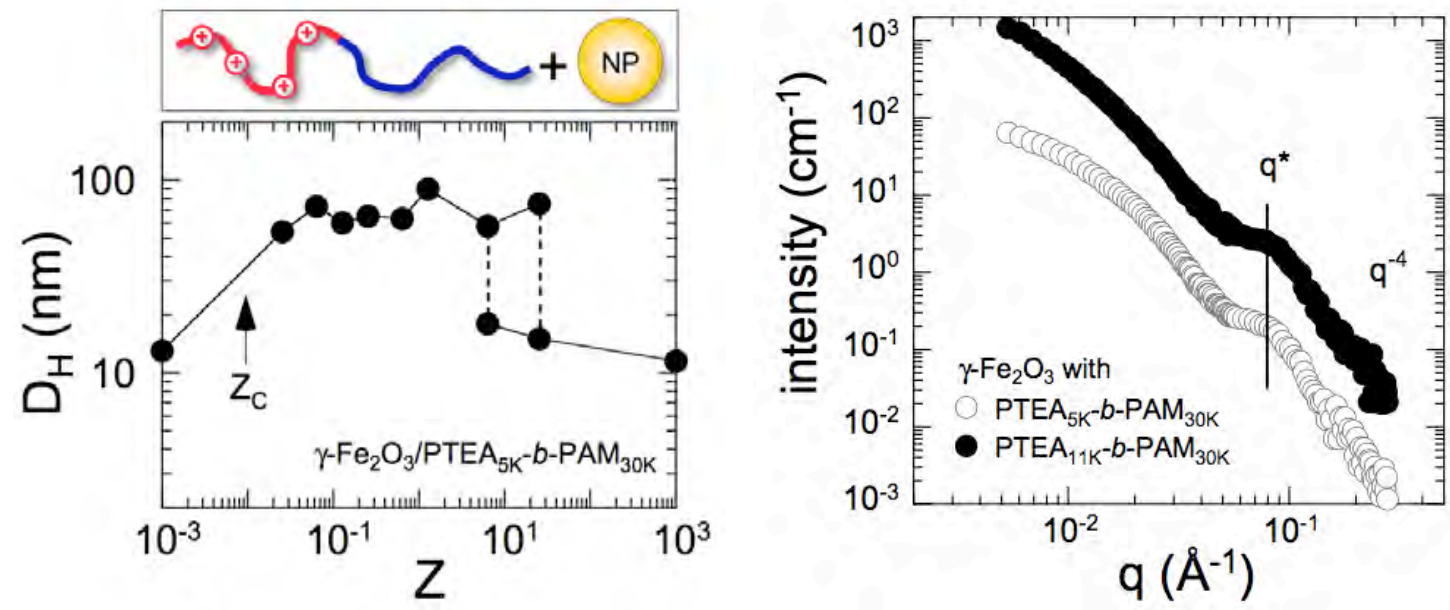

Figure 7 (left) : Hydrodynamic diameter $D_{H}$ as function of the charge ratio $Z$ for mixed dispersions made from citrate-coated iron oxide nanoparticles ( $\mathrm{Cit}-\gamma-\mathrm{Fe}_{2} \mathrm{O}_{3}$ ) complexed with cationic-neutral $\left(P T E A_{5 K}-b-P A M_{30 K}\right)$ block copolymers [90]. With a charge density of $-2 e \mathrm{~nm}^{-2}$, the structural charges of the particles was estimated at 250 elementary charges. The prominent features here are the existence of critical charge ratio $Z_{C}$ around $10^{-2}$ (arrow), a constant hydrodynamic diameter over more than 3 decades in $Z$, and the coexistence state between free and associated particles for $Z>10$ (dashed lines). The panel above the figure illustrates the system considered in Section III for Figs. 7 to 9.

Figure 8 (right) : Scattering intensities for citrate-coated iron oxide nanoparticles complexed with $P T E A_{5 K}-b-P A M_{30 K}$ and with PTEA $A_{11 K}-b-P A M_{30 K}$ block copolymers [95]. Experiments were carried out at $c=2$ wt. \% and in $\mathrm{H}_{2} \mathrm{O}$ for contrast reasons. The intensity for $\mathrm{Cit}-\gamma-\mathrm{Fe}_{2} \mathrm{O}_{3} / \mathrm{PTEA}_{11 \mathrm{~K}}-b-\mathrm{PAM} \mathrm{M}_{30 \mathrm{~K}}$ was shifted by a factor 10 for sake of clarity. The structure peaks observed at high wave-vector at $q^{*}=$ $0.084 A^{-1}$ were interpreted as the signature of dense micro-separated phases of particles. 


\section{III.3 - Iron oxide supracolloidal assembly}

Iron oxide nanoparticles were complexed using poly(trimethylammonium ethylacrylate)- $b$ poly(acrylamide), a diblock that was synthesized by controlled radical polymerization [70, 111]. The molecular weights were $5000 \mathrm{~g} \mathrm{~mol}^{-1}$ and $11000 \mathrm{~g} \mathrm{~mol}^{-1}$ for the charged blocks and $30000 \mathrm{~g} \mathrm{~mol}^{-1}$ for the neutral chain. The polymers were abbreviated $\mathrm{PTEA}_{5 \mathrm{~K}}-b-\mathrm{PAM}_{30 \mathrm{~K}}$ and $\mathrm{PTEA}_{11 \mathrm{~K}}-b-\mathrm{PAM}_{30 \mathrm{~K}}$. Dynamic light scattering performed on Cit $-\gamma-\mathrm{Fe}_{2} \mathrm{O}_{3} / \mathrm{PTEA}_{5 \mathrm{~K}}-b-\mathrm{PAM}_{30 \mathrm{~K}}$ mixed solutions at $\mathrm{c}=0.2 \mathrm{wt}$. \% revealed the presence of one or two diffusive relaxation modes. Fig. 7 displays the evolution of the hydrodynamic diameters $\mathrm{D}_{\mathrm{H}}$ determined for these two modes as a function of the charge ratio. For $\mathrm{Z}$ above a critical value $Z_{C}$, the $D_{H}$ 's were larger than those of the polymers and nanoparticles, and remained at a constant value of $65 \pm 10 \mathrm{~nm}$. For $Z>10$, a second mode associated to the single nanoparticles became apparent.

The results of Fig. 7 bear a strong resemblance to the behavior of surfactant-based complexes. Both systems are characterized by the existence of a critical charge ratio $Z_{C}$ and by a constant diameter above $\mathrm{Z}_{\mathrm{C}}$. The findings of a constant hydrodynamic diameter over more than 3 decades in $\mathrm{Z}$, as well as of coexistence between free and associated particles suggested moreover the existence of a fixed stoichiometry between the charges borne by the polymers and by the particles. Using a stoichiometric model to account for the light scattering results, it was found that the numbers of polymers per particle were 14 for $\mathrm{PTEA}_{5 \mathrm{~K}}-b-\mathrm{PAM}_{30 \mathrm{~K}}$ and 6 for $\mathrm{PTEA}_{11 \mathrm{~K}}-b-\mathrm{PAM}_{30 \mathrm{~K}}$ in the mixed aggregates [90].

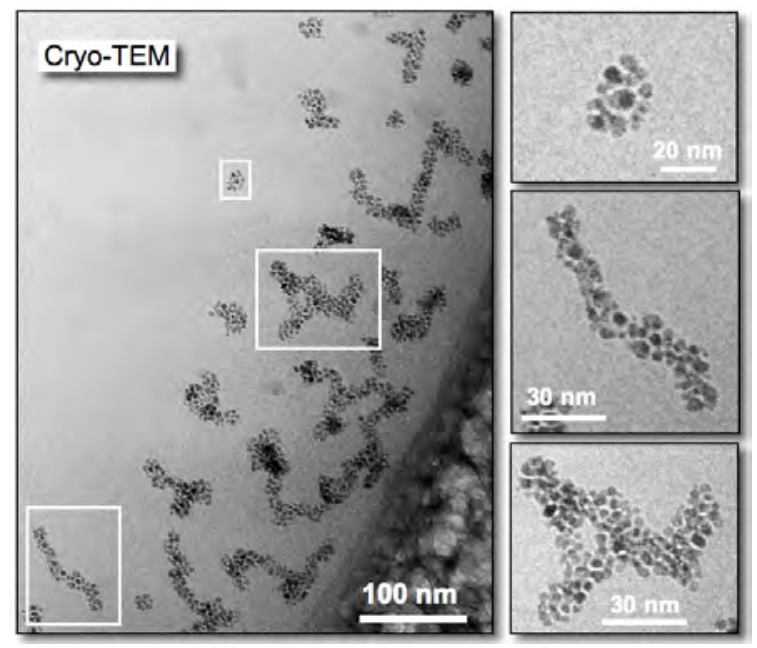

Figure 9 : Cryogenic transmission electron microscopy (Cryo-TEM) images of nanoparticles clusters obtained by complexation between PTEA $A_{11 K}-b-P A M_{30 K}$ and $C i t-\gamma-\mathrm{Fe}_{2} \mathrm{O}_{3}$. The aggregates were obtained according to the direct mixing technique. Their sizes vary from 20 to $200 \mathrm{~nm}$ [99, 95]. Inset : zoom of aggregates showing spherical, linear and branched morphologies.

The analogy with the surfactant complexes was also evidenced using small-angle neutron scattering [95]. For SANS, organic-inorganic hybrids were prepared in $\mathrm{H}_{2} \mathrm{O}$ because the scattering contrast was maximum. Fig. 8 compares the scattering intensisties for aggregates obtained by complexation between Cit $-\gamma-\mathrm{Fe}_{2} \mathrm{O}_{3}$ particles with $\mathrm{PTEA}_{5 \mathrm{~K}}-b-\mathrm{PAM}_{30 \mathrm{~K}}$ and $\mathrm{PTEA}_{11 \mathrm{~K}}-b-\mathrm{PAM}_{30 \mathrm{~K}}$ block copolymers. Two of the three scattering features underscored for the surfactant-based complexes were again observed, namely the strong forward scattering (Point 1) and the structure peak at high wave-vector (Point 3). In Fig. 8, the structure peak was located at $\mathrm{q}^{*}=0.084 \AA^{-1}$, corresponding to an interparticle distance $6.6 \mathrm{~nm}$ and a volume fraction of 0.38 inside the clusters. These later values were derived from Reverse Monte Carlo simulations [95]. Note that because of the intrinsic polydispersity of $\gamma-\mathrm{Fe}_{2} \mathrm{O}_{3}$ particles, the expression $2 \pi / q^{*}$ could not be employed here to calculate the particle-particle distance in the clusters [112]. One characteristic feature was however missing for the $\mathrm{Cit}-\gamma-\mathrm{Fe}_{2} \mathrm{O}_{3} / \mathrm{PTEA}-b$-PAM hybrids, namely the oscillation at intermediate wave-vector. For the surfactants, the oscillation was ascribed to the narrow 
dispersity of the core size distribution. Transmission electron microscopy experiments were then conducted to confirm the SANS data, and thanks to the strong electronic contrast of iron, the entire aggregate morphology and inner structure were observed. Fig. 9 displays aggregates made by direct mixing of $\mathrm{Cit}-\gamma-\mathrm{Fe}_{2} \mathrm{O}_{3}$ and $\mathrm{PTEA}_{11 \mathrm{~K}}-b-\mathrm{PAM}_{30 \mathrm{~K}}$. Covering a spatial field of $0.42 \times 0.56 \mu \mathrm{m}^{2}$, the main frame in Fig. 9 displays well-disperse nanoparticle clusters. However, as anticipated from SANS, the aggregates were polydisperse in shape and in size. The right-hand side insets aim to illustrate the existence of spherical, linear and branched clusters, with dimensions varying from $20 \mathrm{~nm}$ to $200 \mathrm{~nm}$ [99, 95]. Similar findings were found out with non-magnetic $7 \mathrm{~nm}$ citrate-coated cerium oxide nanoparticles, indicating that the anisotropic structures of Fig. 9 did not stem from magnetic dipolar interactions [96, 98]. In most cases with inorganic nanoparticles [98], the same conclusions were reached. Even with an appropriate choice of polymer architecture, the direct mixing strategy was not satisfactory to control the formation of the co-assemblies, as it was the case for the surfactant micelles. Possible explanations for these behaviors are the large number of total charges involved in the process, or the fast kinetics of association between species [98].

\section{IV - The desalting transition : towards the fabrication of controlled size clusters and rods}

\section{IV.1 - Alternative protocols for mixing species with opposite charges}

The protocols for mixing oppositely charged species described in this section were inspired by molecular biology and were developed for the in vitro reconstitutions of chromatin. Chromatin stands for the DNA / histones macromolecular substance that forms the chromosomes of our cells [113]. The protocols applied here consisted first in the screening of the electrostatic interactions by bringing the dispersions of oppositely charged species to high salt concentration, and second in removing the salt progressively by dialysis or by dilution [114]. With this technique, the oppositely charged species were intimately mixed in solution but did not interact owing to the electrostatic screening. Compared to the direct mixing or titration methods introduced in the previous sections, these formulation processes revealed remarkable features such as the occurrence of an abrupt transition between a disperse and an aggregated state of particles.

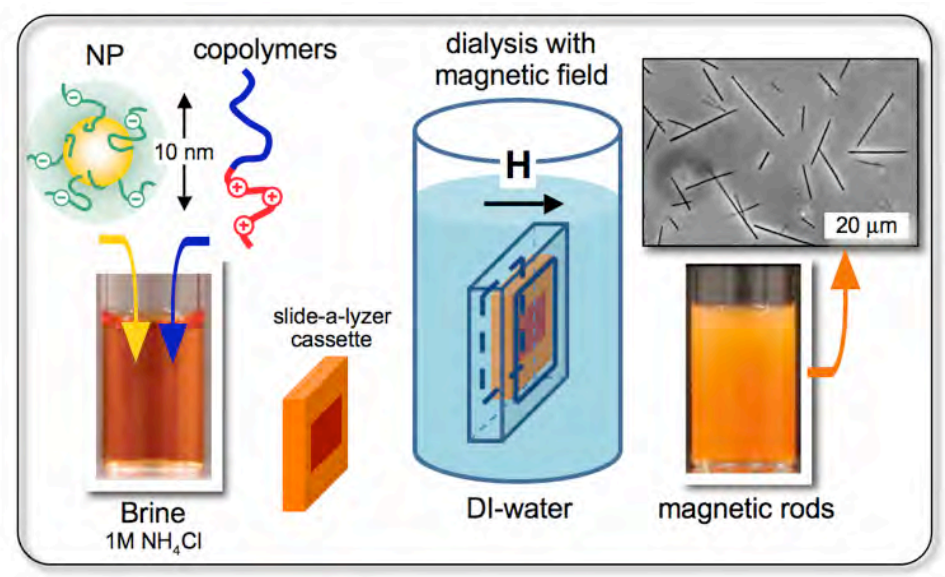

Figure 10 : Schematic representation of the protocol that controls the nanoparticle co-assembly and rod formation [101].

The dialysis or dilution strategies involved in a first step the preparation of separate $1 \mathrm{M}$ salted solutions containing respectively the anionic particles and the cationic-neutral diblock copolymers. In order to improve their stability, the $\gamma-\mathrm{Fe}_{2} \mathrm{O}_{3}$ particles were coated by poly(acrylic acid) with molecular weight 
$2000 \mathrm{~g} \mathrm{~mol}^{-1}$ instead of citrate ligands as in the previous section $[115,100]$. The thickness of the $\mathrm{PAA}_{2 \mathrm{~K}}$ brush tethered on the surface was estimated at $3 \mathrm{~nm}$ by dynamical light scattering. The salt used in the present work was ammonium chloride $\left(\mathrm{NH}_{4} \mathrm{Cl}\right)$. The two solutions were then mixed with each other and it was verified by light scattering that the colloidal stability was not altered by the excess of salt. In a second step, the ionic strength of the dispersions was progressively diminished, by dialysis or by dilution. Dialysis was performed against deionized water using a Slide-a-Lyzer ${ }^{\circledR}$ cassette with molecular weight cut-off $10000 \mathrm{~g} \mathrm{~mol}^{-1}$. The time evolution of the ionic strength was monitored by the measurement of the electric conductivity of the bath. Dialysis was carried under two different conditions, with or without magnetic field. The cartoon in Fig. 10 represents the case where the magnetic field $(0.1 \mathrm{~T})$ was on [116]. In the dilution process, deionized water was added stepwise to the nanoparticle and polymer salted dispersion, at a flow rate that was later translated into a desalting rate $\mathrm{dI} \mathrm{S}_{\mathrm{S}} / \mathrm{dt}$, where $\mathrm{I}_{\mathrm{S}}$ denotes the ionic strength. With dilution, it was possible to vary $\mathrm{dI}_{\mathrm{S}} / \mathrm{dt}^{-}$from $10^{-5}$ to $1 \mathrm{M}$ $\mathrm{s}^{-1}$. With dialysis, the average rate of ionic strength change was of the order of $10^{-3}-10^{-4} \mathrm{M} \mathrm{s}^{-1}$ and the particle concentration remained practically constant.

\section{IV.2 - Evidence of a desalting transition as a function of ionic strength}

Fig. 11 illustrates the light scattering results obtained by dilution using $\mathrm{PAA}_{2 \mathrm{~K}}$-coated $\gamma-\mathrm{Fe}_{2} \mathrm{O}_{3}$ nanoparticles and $\mathrm{PTEA}_{11 \mathrm{~K}}-b-\mathrm{PAM}_{30 \mathrm{~K}}$ copolymers, with $\mathrm{c}=0.2 \mathrm{wt} . \%$ and charge ratio 1 . Dynamic light scattering was monitored on a Brookhaven spectrometer (BI-9000AT) and on a NanoZS (Malvern Instrument) for measurements of the diffusion constant, and from which $\mathrm{D}_{\mathrm{H}}$ is derived [90]. The autocorrelation functions of the scattered light were interpreted using both cumulants and CONTIN procedure provided by the instrument software. After each step addition of de-ionized water, the scattering intensity and hydrodynamic diameter were measured. Doing so, the ionic strength was decreased at a rate $\mathrm{dI}_{\mathrm{S}} / \mathrm{dt} \sim 10^{-4} \mathrm{M} \mathrm{s}^{-1}$ comparable to that of dialysis. For the dispersion containing nanoparticles and copolymers (closed symbols), the data showed an abrupt upturn of the particle sizes at the critical ionic strength $0.39 \mathrm{M}$. This was not the case for the nanoparticles alone (open symbols), for which $\mathrm{D}_{\mathrm{H}}$ remained at the same level $(20 \mathrm{~nm})$. The transition between an unassociated and a clustered state was dubbed desalting transition because it occurred as the excess inorganic salt was removed from the dispersion $[98,116]$.
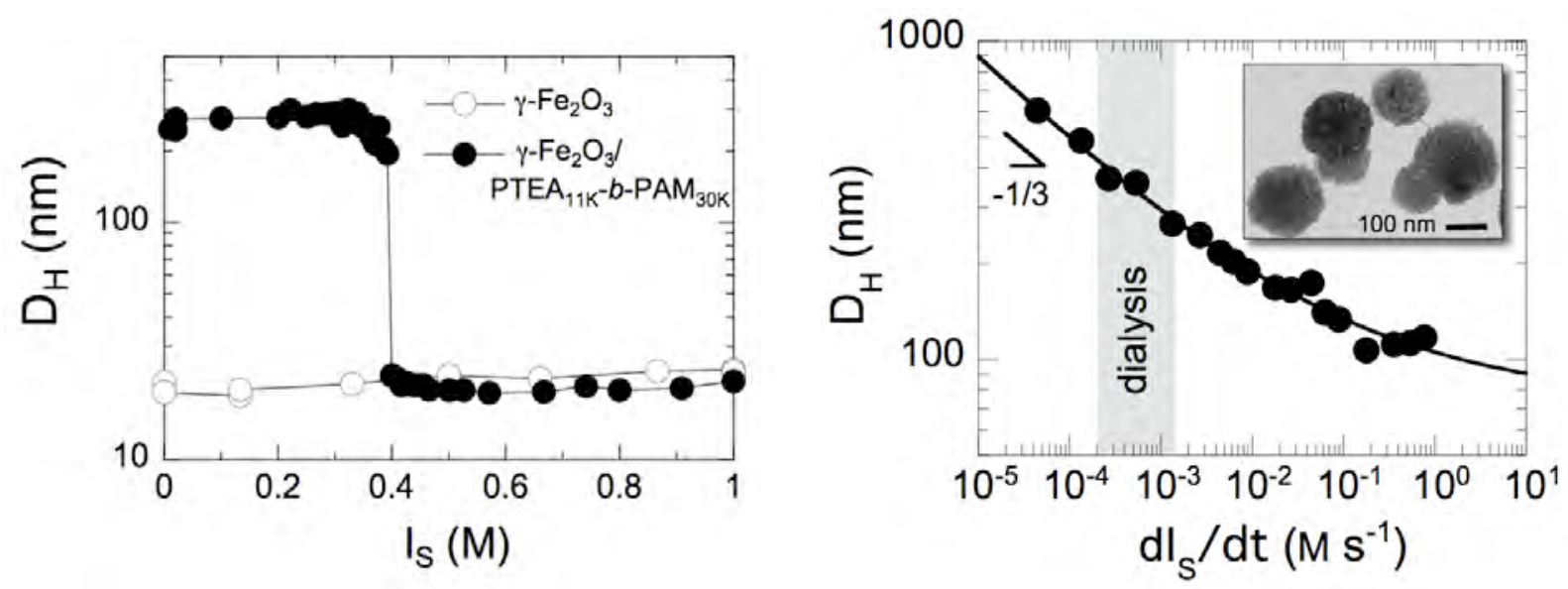

Figure 11 (left) : Ionic strength $I_{S}$ dependence of the hydrodynamic diameter $D_{H}$ for $\mathrm{PAA}_{2 \mathrm{~K}}-\gamma-\mathrm{Fe}_{2} \mathrm{O}_{3}$ nanoparticles (empty symbols) and for a dispersion containing both $\mathrm{PAA}_{2 \mathrm{~K}}-\gamma-\mathrm{Fe}_{2} \mathrm{O}_{3}$ particles and $P T E A_{11 K}-b-P A M_{30 K}$ block copolymers (closed symbols). With decreasing $I_{S}$, an abrupt transition was observed at the critical value of $0.40 \mathrm{M}$ [101]. The transition between the disperse and clustered state of nanoparticles resulted from the combined processes of electrostatic complexation of the cationic blocks with the particles and of slow nucleation and growth of aggregates.

Figure 12 (right) : Hydrodynamic diameters $D_{H}$ as a function of the desalting rate $d I_{S} / d t$ obtained via the dilution and dialysis protocols. Each data point represents a dispersion that underwent the desalting process illustrated in Fig. 11. At low desalting rates, $D_{H}$ displayed an asymptotic scaling of the form $D_{H}$ 
$\sim\left(d I_{S} / d t\right)^{-1 / 3}$. Inset : TEM images of spherical aggregates obtained by dialysis. The aggregate size was estimated at $180 \mathrm{~nm}$.

In a second series of experiments, the desalting transition was monitored on a broad range of desalting rates, between $10^{-5}$ and $1 \mathrm{M} \mathrm{s}^{-1}$. In Fig. 12, each data point represents the hydrodynamic diameter obtained from a dispersion that underwent this desalting process. Interestingly, the critical ionic strength did not depend on the desalting rate. For fast dilution, or quench $\left(\mathrm{dI}_{\mathrm{S}} / \mathrm{dt} \sim 1 \mathrm{M} \mathrm{s}^{-1}\right)$, the aggregates were found around $100 \mathrm{~nm}$. The $\mathrm{D}_{\mathrm{H}}$-values in the fast dilution regime coincide well with those found by direct mixing, suggesting that in terms of kinetics, the mixing of oppositely charged species is equivalent to a quench [98]. With decreasing $\mathrm{dI}_{S} / \mathrm{dt}, \mathrm{D}_{\mathrm{H}}$ increased and displayed an asymptotic scaling law of the form $\mathrm{D}_{\mathrm{H}} \sim \mathrm{dI}_{\mathrm{S}} / \mathrm{dt}^{-1 / 3}$. A power law with exponent $-1 / 3$ indicates that at infinitely slow dilution, the size of the clusters would diverge, the dispersion exhibiting then a macroscopic phase separation. This phase separation was observed by quenching the dispersion slightly below the critical ionic strength [98]. The inset of Fig. 12 displays a TEM image of aggregates obtained by dialysis. The aggregates of average diameter $180 \mathrm{~nm}$ were described as latex-type or composite colloids with a high load of magnetic particles. The volume fraction of magnetic materials was estimated around 0.25 inside the large spheres [95]. The temporal stability of the magnetic clusters was attributed to the fact that the structures formed were non-equilibrium structures.

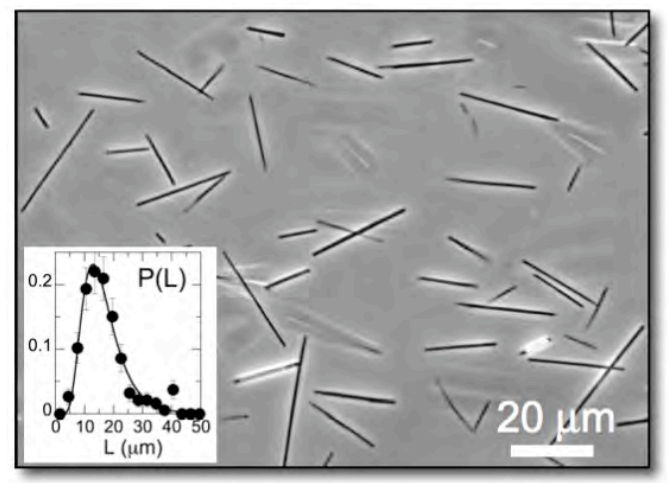

Figure 13 : Phase contrast optical microscopy images (X40) of a nanorod dispersion [116]. Inset : Length distribution of rods obtained with $7.7 \mathrm{~nm} P A A_{2 K}-\gamma-\mathrm{Fe}_{2} \mathrm{O}_{3}$ particles. The continuous line was derived from best fit calculations using a log-normal distribution, with median length $\mathrm{L}_{0}^{\text {rod }}=15 \mu \mathrm{m}$ and polydispersity $\mathrm{s}^{\mathrm{rod}}=0.50$. The polydispersity $\mathrm{s}^{\mathrm{rod}}$ was defined as the ratio between the standard deviation and the average.

\section{IV.3 - Magnetic clusters and rods}

In a third series of experiments, dialysis of mixed salted solutions was operated under a constant magnetic field of $0.1 \mathrm{~T}$, i.e. corresponding to the set-up of Fig. 10. Once the ionic strength of the dialysis bath reached its stationary value, the magnetic field was removed and the solutions were studied by optical microscopy. Fig. 13 shows an optical transmission microscopy image of rods made of $\mathrm{D}_{0}^{\mathrm{NP}}=$ $7.7 \mathrm{~nm} \gamma-\mathrm{Fe}_{2} \mathrm{O}_{3}$ particles at concentration $\mathrm{c}=0.1 \mathrm{wt}$. $\%$ and charge ratio about $1[101,102,104]$. In the absence of magnetic field, anisotropic structures with random orientations and lengths comprised between 1 and $50 \mu \mathrm{m}$ were clearly seen. Series of images similar to Fig. 13 were analyzed quantitatively to retrieve the rod length distribution. It was found to be well accounted for by a log-normal function with median length $\mathrm{L}_{0}^{\text {rod }}(=15 \mu \mathrm{m})$ and polydispersity $\mathrm{s}^{\text {rod }}(=0.5)$, as shown by the inset in Fig. 13 . The nanostructured rods are defined as higher level objects because their construct involves three different length scales : $i$ ) the size of the elementary particles, around $10 \mathrm{~nm}$, ii) the diameter of the rod which found between 200 and $500 \mathrm{~nm}$ and iii) the length of the rods comprised between 1 and $50 \mu \mathrm{m}$. 


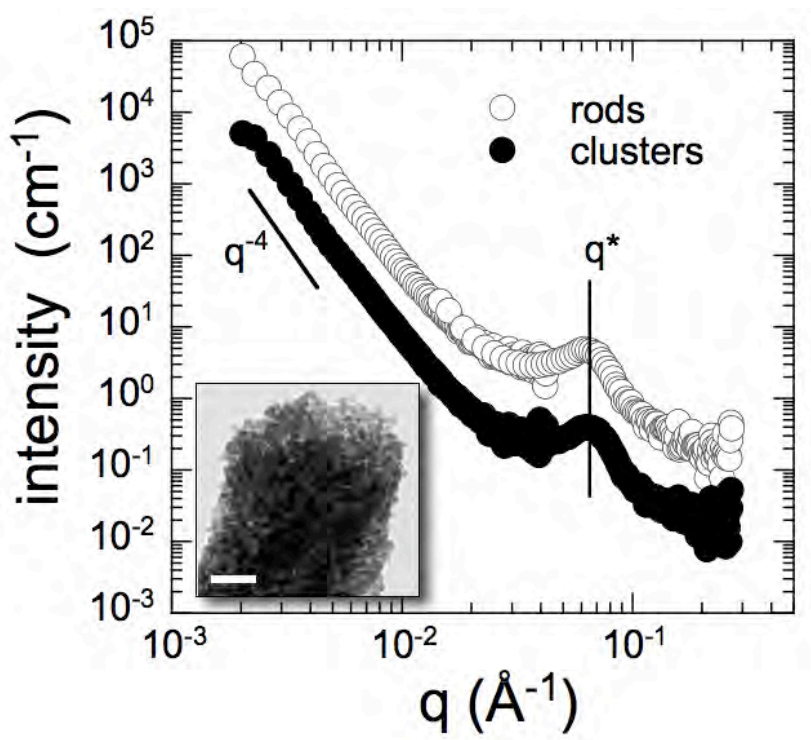

Figure 14 : Neutron intensities of clusters $\left(D_{H}=310 \mathrm{~nm}\right)$ and rods $\left(\mathrm{L}_{0}^{\text {rod }}=15 \mu \mathrm{m}\right)$ obtained by electrostatic complexation between $P A A_{2 K}-\gamma-F_{2} O_{3}$ nanoparticles and $P T E A_{11 K}-b-P A M_{30 K}$ block copolymers through the dialysis technique. The data for the rods were shifted by a factor 10 for clarity. The structure peaks observed at $q^{*}=0.064 A^{-1}$ were interpreted as the signature of a dense packing of the particles within the supracolloidal objects. Inset : TEM image of the extremity of a rod [104].

If a magnet was brought near to the sample, the rods reoriented spontaneously and followed the magnetic field lines. The coupling between the rods and the external field was shown to originate from the superparamagnetic properties of the elongated structures, a property that could be exploited in microfluidics and in therapeutics for the elaboration of micro-actuators [117]. Based on a comprehensive study of the morphology diagram of the rods, we elaborated on the mechanisms of rod formation. According to Ref. [116], the mechanism proceeds in two steps : first the formation and growth of spherical clusters of particles, and then the alignment of the clusters induced by the magnetic dipolar interactions. As far as the kinetics of the two processes is concerned, the clusters growth and their alignment occur concomitantly, leading to a continuous accretion of particles or small clusters, and a welding of the rodlike structure.

To finally demonstrate that the rods were nanostructured, small-angle neutron scattering was performed on dispersions prepared in $\mathrm{H}_{2} \mathrm{O}$, again for contrast reasons. The aggregates were fabricated by dialysis according to the protocol of Fig. 10 and using $\mathrm{PAA}_{2 \mathrm{~K}}-\gamma-\mathrm{Fe}_{2} \mathrm{O}_{3}$ nanoparticles of diameter $\mathrm{D}_{0}^{\mathrm{NP}}=7.7 \mathrm{~nm}$ and polydispersity $\mathrm{s}^{\mathrm{NP}}=0.22$ [116]. The clusters had an hydrodynamic diameter of $310 \mathrm{~nm}$ and the rods a median length of $\mathrm{L}_{0}^{\text {rod }}=15 \mu \mathrm{m}$, with a polydispersity of 0.50 . Fig. 14 shows that the scattering intensities for the two samples were very similar. The data for the rods were shifted by a factor 10 for clarity. From the three characteristics found for the surfactant-based complexes, only the structure peak at large wave-vectors, here located at $\mathrm{q}^{*}=0.064 \AA^{-1}$ remained (Point 3). The two structure peaks are more intense than those of Fig. 8. This findings is in agreement with the fact that the scattering objects are now in the micrometer range. The low q-region exhibited typical Porod $\mathrm{q}^{-4}$-dependences characteristic for large objects with abrupt interfaces. In the inset of Fig. 14, a TEM image of the extremity of a rod is illustrated, corroborating the dense packing of the particles in the cylindrical body. At the application of a constant magnetic field of strength $10 \mathrm{mT}$, the rods reoriented. Depending on their orientations with respect to the incoming beam, the scattered intensity collected on a twodimensional detector remained isotropic or became strongly anisotropic (Fig. 15). In Fig. 15a, the magnetic field was parallel to the neutron beam and in the configuration of the spectrometer, the dispersion appeared isotropic to the incoming neutron $[118,119]$. In contrast, when the field was in the 
plane of the detector as in Fig. 15b and 15c, the scattering intensity was enhanced in the reciprocal space in the directions perpendicular to the rods. In agreement with optical microscopy results, these findings confirmed that the basic structure of the rods was not altered by the application of a field, only their reorientations were modified.

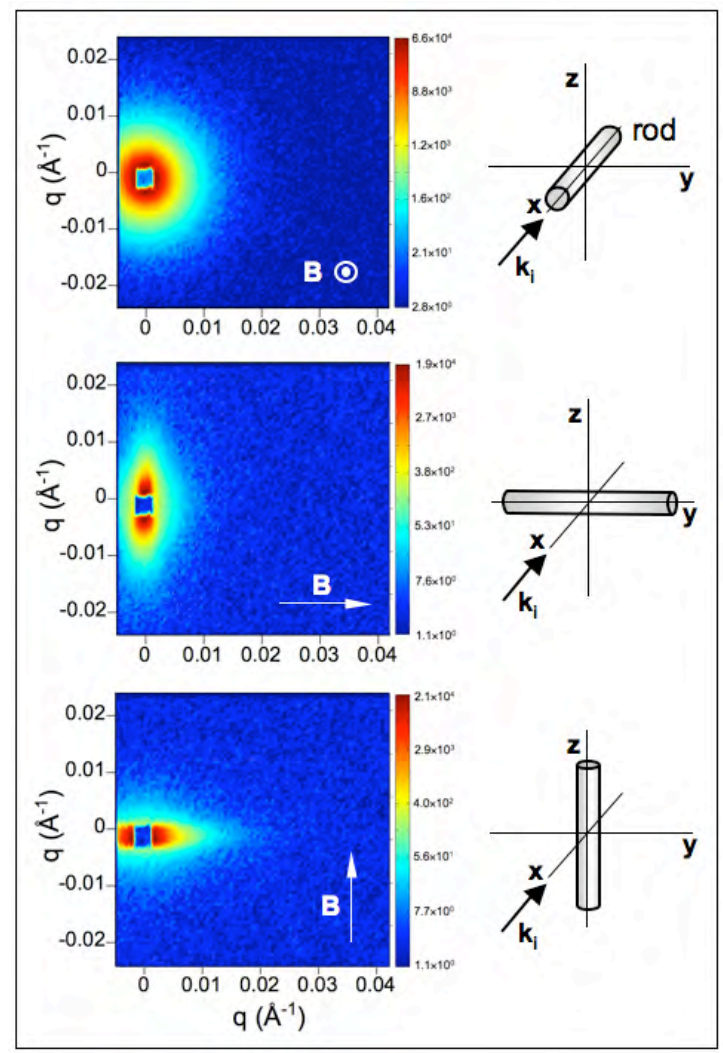

Figure 15 : Two-dimensional scattering intensities obtained from iron oxide-based nanostructured rods. The nanorods were oriented by the application of a field of amplitude $B=10 \mathrm{mT}:$ a) $\boldsymbol{B} / / \boldsymbol{k}_{\boldsymbol{i}} ; \boldsymbol{b}$ and c) $\boldsymbol{B} \perp \boldsymbol{k}_{\boldsymbol{i}}$, where $\boldsymbol{k}_{\boldsymbol{i}}$ denotes the wave-vector of the incident neutron beam.

\section{V - Conclusion}

In the preceding sections, we have shown that nanocolloids such as surfactant micelles and iron oxide magnetic nanoparticles share similar properties when mixed with ionic-neutral diblock copolymers : above a critical charge ratio, core-shell nanostructures form spontaneously under various mixing conditions. The driving forces for the formation of higher-level objects are insured by electrostatic attractions between the opposite charges, as well as by the release of the condensed counterions. In the following, we recapitulate the milestones that are important in the present experimental context, and suggest some applications for these nanostructured materials.

Our initial approach using diblock copolymers and surfactants was to design hierarchical aggregates of different sizes and morphologies by adjusting the architecture of the polymers. The goal was to assess the analogy between electrostatic complexes and polymeric micelles made from amphiphilic copolymers. For that, we studied synthetic copolymers with various architectures, including diblock, triblock, graft and star structures and of various molecular weights between 1000 to $100000 \mathrm{~g} \mathrm{~mol}^{-1}$. The results of this strategy was very successful. On the two surfactant/copolymer systems investigated, DTAB/PAA- $b$-PAM and sodium dodecyl sulfate/PTEA- $b$-PAM $[35,37,38]$, it was possible to produce aggregates of sizes lying between 20 and $500 \mathrm{~nm}$. By changing the formulation conditions it was also possible to trigger morphologies going from spherical and cylindrical structures [77]. From these 
studies, it became clear however that electrostatic complexes depended on the preparation and mixing conditions, such as the $\mathrm{pH}$, concentration, mixing speed etc.... We mentioned the case of $\mathrm{DTAB} / \mathrm{PANa}_{6.5 \mathrm{~K}}-b-\mathrm{PAM}_{37 \mathrm{~K}}$ samples having been made at the same concentration, but using different pathways [22]. Their structures were still core-shell, but their neutron signatures indicated average cores of 22 and $47 \mathrm{~nm}$ respectively, with no evidence whatsoever of a late evolution of their internal structures. The same was observed with inorganic nanoparticles $[99,95]$. These findings prompted us to search for alternative approaches, and in particular to look for processes where the complexation would be kinetically controlled instead of chemically controlled.

Many different techniques were already used to control the self- and co-assembly of macromolecular objects under strong attractive interactions. This includes confined impinging jets mixers [120] or microfluidic lab-on-a-chip devices [121]. In this work, we adopt a strategy borrowed from molecular biology for the in vitro preparation of chromatin. It is demonstrated that playing with the desalting rate allowed us to fine-tune the kinetics of association in a very accurate way. By varying the desalting rate from 1 to $10^{-5} \mathrm{M} \mathrm{s}^{-1}$, nanoparticle clusters with average sizes $100-1000 \mathrm{~nm}$ were fabricated.

The enhanced stability of the nanostructures built up with surfactants and with inorganic nanoparticles is interpreted as resulting from a non-equilibrium associating process. Once the aggregates are formed, their microstructure remained unaltered over periods longer than years. The non-equilibrium character of these objects shows up with the nanorods made from sub-10 nm magnetic nanoparticles. The rods can be moved in the solvent or rotated by the application of a field or gradient without changing their internal structure, as shown by neutron scattering. Because the present strategy for electrostatic coassembly of nanocolloids is simple and versatile, it should open new perspectives for the design of nanodevices, such as tips, tweezers, actuators etc., applicable in biophysics and biomedicine; for stimulating and sorting living cells; or as novel contrast agents for the drug delivery.

Acknowledgement : The present review would not have been possible without the extended network of colleagues who participated to this research. It is a pleasure to acknowledge here the collaborations I had over the years with Jean-Christophe Castaing, Jean-Paul Chapel, Galder Cristobal, Jérôme Fresnais, Bruno Frka-Petesic, Pascal Hervé, Eléna Ishow, Mikel Morvan, Julian Oberdisse, Régine Perzynski, Ling Qi, Olivier Sandre, Amit Sehgal, Minhao Yan and Kazuhiko Yokota. I would also like to thank Bernard Cabane, Valérie Cabuil, Andrejs Cebers, Martien Cohen-Stuart, Mathias Destarac, Eric Kaler, Christophe Lavelle, Sébastien Lecommandoux, Patrick Maestro, Nathalie Mignet, Lennard Picullel, Didier Roux, Serge Stoll for fruitful discussions. The Laboratoire Léon Brillouin in Saclay (Annie Brûlet, Fabrice Cousin) and the Institute Laue-Langevin in Grenoble (Isabelle Grillo, Ralph Schweins) are acknowledged for their technical and financial support. I would like to thank the Complex Fluids Laboratory (UMR Rhodia-CNRS n ${ }^{\circ} 166$ ) and the Laboratoire Physico-chimie des Electrolytes, Colloïdes et Sciences Analytiques (UMR Université Pierre et Marie Curie-CNRS n ${ }^{\circ}$ 7612) for our long-lasting collaborations and the later for providing us with the magnetic nanoparticles. During the last decade, this research work was supported in part by Rhodia (France), by the Agence Nationale de la Recherche under the contracts BLAN07-3_206866 and ANR-09-NANO-P200-36, by the University Denis Diderot Paris 7 (BQR2006), by the European Community through the project : "NANO3T-Biofunctionalized Metal and Magnetic Nanoparticles for Targeted Tumor Therapy", project number 214137 (FP7-NMP2007-SMALL-1) and by the Région Ile-de-France in the DIM framework related to Health, Environnement and Toxicology (SEnT).

\section{References}

[1] Min YJ, Akbulut M, Kristiansen K, Golan Y, Israelachvili J. The role of interparticle and external forces in nanoparticle assembly. Nat Mater. 2008;7:527-38.

[2] Mann S. Self-assembly and transformation of hybrid nano-objects and nanostructures under equilibrium and non-equilibrium conditions. Nat Mater. 2009;8:781-92. 
[3] Naka K, Chujo Y. Nanohybridized Synthesis of Metal Nanoparticles and Their Organization. Nanohybridization of Organic-Inorganic Materials2009. p. 3-40.

[4] Voets IK, de Keizer A, Cohen Stuart MA. Complex coacervate core micelles. Adv Colloid Interface Sci. 2009;147-148:300-18.

[5] Lusser A, Kadonaga JT. Strategies for the reconstitution of chromatin. Nature Methods. 2004;1:19-26.

[6] Schiessel H. The Physics of Chromatin. J Phys: Condens Matter. 2003;15:R699 - R774.

[7] Korolev N, Vorontsova OV, Nordenskiold L. Physicochemical analysis of electrostatic foundation for DNAprotein interactions in chromatin transformations. Progress in Biophysics \& Molecular Biology. 2007;95:23-49.

[8] Decher G. Fuzzy Nanoassemblies: Toward Layered Polymeric Multicomposites. Science. 1997;277:1232 - 7.

[9] Laugel N, Betscha C, Winterhalter M, Voegel J-C, Schaaf P, Ball V. Relationship between the Growth Regime of Polyelectrolyte Multilayers and the Polyanion/Polycation Complexation Enthalpy. J Phys Chem B. 2006;110:19443 - 9 .

[10] Caruso F, Caruso RA, Möhwald H. Nanoengineering of Organic and Hybrid Hollow Spheres by Colloidal Templating. Science. 1998;282:1111 - 4.

[11] Ladam G, Schaad P, Voegel JC, Schaaf P, Decher G, Cuisinier F. In situ determination of the structural properties of initially deposited polyelectrolyte multilayers. Langmuir. 2000;16:1249-55.

[12] Caruso F, Möhwald H. Preparation and Characterization of Ordered Nanoparticle and Polymer Composite Multilayers on Colloids. Langmuir. 1999;15:8276 - 81.

[13] Ostrander JW, Mamedov AA, Kotov NA. Two Modes of Linear Layer-by-Layer Growth of NanoparticlePolyelectrolyte Multilayers and Different Interactions in the Layer-by-Layer Deposition. J Am Chem Soc. 2001;123:1101 - 10.

[14] Lee SW, Kim B-S, Chen S, Shao-Horn Y, Hammond PT. Layer-by-Layer Assembly of All Carbon Nanotube Ultrathin Films for Electrochemical Applications. Journal of the American Chemical Society. 2009;131:671-9.

[15] Wu ZZ, Walish J, Nolte Z, Rubner MF. Deformable antireflection coatings from polymer and nanoparticle multilayers. Adv Mater. 2006;18:2699.

[16] Lee D, Rubner MF, Cohen RE. All-Nanoparticle Thin-Film Coatings. Nano Letters. 2007;7:1444-.

[17] Harada A, Kataoka K. Formation of polyion complex micelles in an aqueous milieu from a pair of oppositelycharged block-copolymers with poly(ethylene glycol) segments. Macromolecules. 1995;28:5294-9.

[18] Harada A, Kataoka K. Novel Polyion Complex Micelles Entrapping Enzyme Molecules in the Core : Preparation of Narrowly-Distributed Micelles from Lyzosome and Poly(ethylene glycol)-Poly(aspartic acid) Block Copolymers in Aqueous Medium. Macromolecules. 1998;31:288 - 94.

[19] Harada A, Kataoka K. Novel Polyion Complex Micelles Entrapping Enzyme Molecules in the Core : Characterization of the Micelles Prepared at Nonstoechiometric ratios. Langmuir. 1999;15:4208 - 12.

[20] Kataoka K, Harada A, Nagasaki Y. Block Copolymer Micelles for Drug Delivery: Design, Characterisation and Biological Significance. Adv Drug Del Rev. 2001;47:113 - 31.

[21] Bain CD, Claesson PM, Langevin D, Meszaros R, Nylander T, Stubenrauch C, et al. Complexes of surfactants with oppositely charged polymers at surfaces and in bulk. Adv Colloid Interface Sci. 2010;155:32-49.

[22] Berret J-F, Cristobal G, Herve P, Oberdisse J, Grillo I. Structure of colloidal complexes obtained from neutral/polyelectrolyte copolymers and oppositely charged surfactants. European Physical Journal E. 2002;9:30111.

[23] Stuart MAC, Besseling NAM, Fokkink RG. Formation of Micelles with Complex Coacervate Cores. Langmuir. 1998;14:6846 - 9.

[24] Burgh Svd, Keizer Ad, Stuart MAC. Complex Coarcevation Core Micelles. Colloidal Stability and Aggregation Mechanism. Langmuir. 2004;20:1073 - 84.

[25] Stuart MAC, Hofs B, Voets IK, Keizer Ad. Assembly of polyelectrolyte-containing block copolymers in aqueous media. Curr Opin Colloid Interface Sci. 2005;10:30 - 6.

[26] Voets IK, deKeizer A, CohenStuart MA, Justynska J, Schlaad H. Irreversible Structural Transitions in Mixed Micelles of Oppositely Charged Diblock Copolymers in Aqueous Solution. Macromolecules. 2007;40:2158-64.

[27] Voets IK, vanderBurgh S, Farago B, Fokkink R, Kovacevic D, Hellweg T, et al. Electrostatically Driven Coassembly of a Diblock Copolymer and an Oppositely Charged Homopolymer in Aqueous Solutions. Macromolecules. 2007;40:8476-82. 
[28] Jeong JH, Kim SW, Park TG. Novel Intracellular Delivery System of Antisense Oligonucleotide by SelfAssembled Hybrid Micelles Composed of DNA/PEG Conjugate and Cationic Fusogenic Peptide. Bioconjugate Chem. 2003;14:473 - 9.

[29] Raspaud E, Olvera-de-la-Cruz M, Sikorav J-L, Livolant F. Precipitation of DNA by Polyamines: A Polyelectrolyte Behavior. Biophys J. 1998;74:381 - 93.

[30] Raspaud E, Chaperon I, Leforestier A, Livolant F. Spermine-Induced Aggregation of DNA, Nucleosome, and Chromatin. Biophys J. 1999;77:1547 - 55.

[31] Bronich TK, Kabanov AV, Kabanov VA, Yui K, Eisenberg A. Soluble Complexes from Poly(ethylene oxide)-block-Polymethacrylate Anions and N-alkylpyridinium Cations. Macromolecules. 1997;30:3519 - 25.

[32] Bronich TK, Cherry T, Vinogradov S, Eisenberg A, Kabanov VA, Kabanov AV. Self-assembly in mixtures of Poly(ethylene oxide)-graft-Poly(ethyleneimine) and alkyl sulfates. Langmuir. 1998;14:6101 - 6.

[33] Bronich TK, Popov AM, Eisenberg A, Kabanov VA, Kabanov AV. Effects of Block Length and Structure of Siurfactant on Self-Assembly and Solution Behavior of Block Ionomer Complexes. Langmuir. 2000;16:481 - 9.

[34] Herve P, Destarac M, Berret J-F, Lal J, Oberdisse J, Grillo I. Novel core-shell structure for colloids made of neutral/polyelectrolyte diblock copolymers and oppositely charged surfactants. Europhysics Letters. 2002;58:9128 .

[35] Berret J-F, Herve P, Aguerre-Chariol O, Oberdisse J. Colloidal complexes obtained from charged block copolymers and surfactants: A comparison between small-angle neutron scattering, Cryo-TEM, and simulations. J Phys Chem B. 2003;107:8111-8.

[36] Solomatin SV, Bronich TK, Bargar TW, Eisenberg A, Kabanov VA, Kabanov AV. Environmentally responsive nanoparticles from block ionomer complexes: Effects of $\mathrm{pH}$ and ionic strength. Langmuir. 2003;19:8069-76.

[37] Berret J-F, Vigolo B, Eng R, Herve P, Grillo I, Yang L. Electrostatic self-assembly of oppositely charged copolymers and surfactants: A light, neutron, and X-ray scattering study. Macromolecules. 2004;37:4922-30.

[38] Berret J-F. Evidence of overcharging in the complexation between oppositely charged polymers and surfactants. J Chem Phys. 2005;123:164703.

[39] Annaka M, Morishita K, Okabe S. Electrostatic self-assembly of neutral and polyelectrolyte block copolymers and oppositely charged surfactant. J Phys Chem B. 2007;111:11700-7.

[40] Sjöström L, Akesson T, Jönsson B. Charge Reversal in Electric Double Layers - A Balance between Energy and Entropy. Ber BunsengesPhys Chem. 1996;100:889 - 93.

[41] Harries D, May S, Gelbart WM, Ben-Shaul A. Structure, Stability, and Thermodynamics of Lamellar DNALipid Complexes. Biophys J. 1998;75:159 - 73.

[42] Kennedy MT, Pozharski EV, Rakhmanova VA, MacDonald RC. Factors Governing the Assembly of Cationic Phospholipid-DNA Complexes. Biophys J. 2000;78:1620 - 33.

[43] Ball V, Winterhalter M, Schwinte P, Lavalle P, Voegel J-C, Schaaf P. Complexation Mechanism of Bovine Serum Albumine and Poly(allylamine hydrochloride). J Phys Chem B. 2002;106:2357 - 64.

[44] Wagner K, Harries D, May S, Kahl V, Rädler JO, Ben-Shaul A. Direct Evidence for Counterion Release upon Cationic Lipid-DNA Condensation. Langmuir. 2000;16:303 - 6.

[45] Ben-Tal N, Honig B, Bagdassarian CK, Ben-Shaul A. Association Entropy in Adsorption Process. Biophys J. 2000;79:1180 - 7 .

[46] Antonietti M, Conrad J. Synthesis of Very Highly Liquid Crystalline Phases by Complex Formation of Polyacrylic Acid with Cationic Surfactants. Angew Chem Int Ed. 1994;33:1869.

[47] Ilekti P, Piculell L, Tournilhac F, Cabane B. How to Concentrate an Aqueous Polyelectrolyte/Surfactant Mixtures by Adding Water. J Phys Chem B. 1998;102:344 - 51.

[48] Svensson A, Piculell L, Cabanne B, Ilekti P. A New Approach to the Phase Behavior of Oppositely Charged Polymers and Surfactants. J Phys Chem B. 2002;106:1013 - 8.

[49] Ha B-Y, Liu AJ. Effect of Non-Pairwise-Additive Interactions on Bundles. Phys Rev Lett. 1998;81:1011 - 4.

[50] Hansson P. Self-Assembly of Ionic Surfactants in Polyelectrolyte Solutions : A Model for Mixtures of Opposite Charge. Langmuir. 2001;17:4167 - 79.

[51] Guillot S, McLoughlin D, Jain N, Delsanti M, Langevin D. Polyelectrolyte-Surfactant Complexes at Interfaces and in Bulk. J Phys: Condens Matter. 2003;15:S219 - S24. 
[52] Weinbreck F, de Vries R, Schrooyen P, de Kruif CG. Complex Coacervation of Whey Proteins and Gum Arabic. Biomacromolecules. 2003;4:293 - 303.

[53] Sinn CG, Dimova R, Antonietti M. Isothermal Titration Calorimetry of the Polyelectrolyte/Water Interaction and Binding of $\mathrm{Ca} 2+$ : Effects Determining the Quality of Polymeric Scale Inhibitors. Macromolecules. 2004;37:3444 - 50.

[54] Thalberg K, Lindman B, Bergfeldt K. Phase Behavior of Systems of Polyacrylate and Cationic Surfactants. Langmuir. 1991;7:2893 - 8.

[55] Wang YL, Kimura K, Huang QR, Dubin PL, Jaeger W. Effects of salt on polyelectrolyte-micelle coacervation. Macromolecules. 1999;32:7128-34.

[56] Pagac ES, Prieve DC, Tilton RD. Kinetics and Mechanism of Cationic Surfactant Adsorption and Coadsorption with Cationic Polyelectrolytes at the Silica-Water Interface. Langmuir. 1998;14:2333 - 42.

[57] Rojas OJ, Claesson PM, Berglund KD, Tilton RD. Coadsorption and Surface Forces for Selective Surfaces in Contact with Aqueous Mixtures of Oppositely Charged Surfactants and Low Charge Density Polyelectrolytes. Langmuir. 2004;20:3221-30.

[58] Naderi A, Claesson PM. Adsorption Properties of Polyelectrolyte-Surfactant Complexes on Hydrophobic Surfaces Studied by QCM-D. Langmuir. 2006;22:7639 - 45.

[59] Kahandurina YV, Dembo AT, Bogacheva VB, Zezin AB, Kabanov VA. Structure of Polycomplexes Composed of Cross-linked Polyacrylate and cationic Micelle-Forming Surfactants. Polymer Science. 1994;36:189 $-94$.

[60] Sokolov E, Yeh F, Khokhlov A, Grinberg VY, Chu B. Nanostructure Formation in Polyelectrolyte-Surfactant Complexes. J Phys Chem B. 1998;102:7091 - 8.

[61] Yeh FJ, Sokolov EL, Walter T, Chu B. Structure studies of poly(diallyldimethylammonium chloride-coacrylamide) gels/sodium dodecyl sulfate complex. Langmuir. 1998;14:4350-8.

[62] Ilekti P, Martin T, Cabanne B, Piculell L. Effects of Polyelectrolytes on the Structures and Interactions of Surfactant Aggregates. J Phys Chem B. 1999;103:9831 - 40.

[63] Zhou S, Yeh F, Burger C, Chu B. Formation and Transitions of Highly Ordered Structures of PolyelectrolyteSurfactant Complexes. J Phys Chem B. 1999;103:2107 - 12.

[64] Wang YL, Kimura K, Dubin PL, Jaeger W. Polyelectrolyte-micelle coacervation: Effects of micelle surface charge density, polymer molecular weight, and polymer/surfactant ratio. Macromolecules. 2000;33:3324-31.

[65] Ashbaugh HS, Lindman B. Swelling and Structural Changes of Oppositely Charged Polyelectrolyte GelMixed Surfactant Complexes. Macromolecules. 2001;34:1522 - 5.

[66] Kogej K, Evmenenko G, Theunissen E, Berghmans H, Reynaers H. Investigation of Structures in Polyelectrolytre/Surfactant Complexes by X-ray Scattering. Langmuir. 2001;17:3175 - 84.

[67] Pergushov DV, Remizova EV, Feldthusen J, Zezin AB, Muller AHE, Kabanov VA. Novel Water-Soluble Micellar Interpolyelectrolyte Complexes. The Journal of Physical Chemistry B. 2003;107:8093-6.

[68] Danial M, Klok H-A, Norde W, CohenStuart MA. Complex Coacervate Core Micelles with a LysozymeModified Corona. Langmuir. 2007;23:8003 - 9.

[69] Bronich TK, Vinogradov SV, Kabanov AV. Interaction of nanosized copolymer networks with oppositely charged amphiphilic molecules. Nano Letters. 2001;1:535-40.

[70] Destarac M, Bzducha W, Taton D, Gauthier-Gillaizeau I, Zard SZ. Zanthates as Chain-Transfer Agents in Controlled Radical Polymerization (MADIX): Structural Effect of the O-Alkyl Group. Macromol Rapid Commun. 2002;23:1049 - 54

[71] McGrath KM. Phase Behavior of Dodecyltrymethylammonium Bromide/Water Mixtures. Langmuir. $1995 ; 11: 1835-9$.

[72] Konop AJ, Colby RH. Role of Condensed Counterions in the Thermodynamics of Surfactant Micelle Formation with and without Oppositely Charged Polyelectrolytes. Langmuir. 1999;15:58 - 65.

[73] Lindner P, Zemb T. Neutrons, X-rays and Light : Scattering Methods Applied to Soft Condensed Matter. Amsterdam: Elsevier; 2002.

[74] Oberdisse J, Demé B. Structure of Latex-Silica Nanocomposite Films : A Small-Angle Neutron Scattering Study. Macromolecules. 2002;35:4397 - 405.

[75] Oberdisse J, Hine P, Pyckhout-Hintzen W. Structure of interacting aggregates of silica nanoparticles in a polymer matrix: small-angle scattering and reverse Monte Carlo simulations. Soft Matter. 2007;3:476 - 85. 
[76] Trabelsi S, Guillot S, Raspaud E, Delsanti M, Langevin D, Boue F. New nano- and microparticles with a liquid-crystal-like interior. Adv Mater. 2006;18:2403-+.

[77] Berret JF. Sphere-to-cylinder transition in hierarchical electrostatic complexes. Colloid and Polymer Science. 2009;287:801-10.

[78] Cousin F, Gummel J, Ung D, Boue F. Polyelectrolyte-Protein Complexes: Structure and Conformation of Each Specie Revealed by SANS. Langmuir. 2005;21:9675 - 88.

[79] Pankhurst QA, Connolly J, Jones SK, Dobson J. Applications of Magnetic Nanoparticles in Biomedicine. J Phys D: Appl Phys. 2003;36:R167 - R81.

[80] Michalet X, Pinaud FF, Bentolila LA, Tsay JM, Doose S, Li JJ, et al. Quantum Dots for Live Cells, in Vivo Imaging, and Diagnostics. Science. 2005;307:538 - 44.

[81] Eustis S, El-Sayed MA. Why gold nanoparticles are more precious than pretty gold: Noble metal surface plasmon resonance and its enhancement of the radiative and nonradiative properties of nanocrystals of different shapes. Chem Soc Rev. 2006;35:209-17.

[82] Boal AK, Ilhan F, DeRouchey JE, Thurn-Albrecht T, Russell TP, Rotello VM. Self-assembly of nanoparticles into structured spherical and network aggregates. Nature. 2000;404:746-8.

[83] Gaponik N, Radtchenko IL, Sukhorukov GB, Rogach AL. Luminescent Polymer Microcapsules Addressable by a Magnetic Field. Langmuir. 2004;20:1449 - 52.

[84] Ewers TD, Sra AK, Norris BC, Cable RE, Cheng C-H, Shantz DF, et al. Spontaneous Hierarchical Assembly of Rhodium Nanoparticles into Spherical Aggregates and Superlattices. Chem Mater. 2005;17:514-20.

[85] Frankamp BL, Boal AK, Tuominen MT, Rotello VM. Direct Control of the Magnetic Interaction between Iron Oxide Nanoparticles through Dendrimer-Mediated Self-Assembly. J Am Chem Soc. 2005;127:9731 - 5.

[86] Osterloh FE, Hiramatsu H, Dumas RK, Liu K. Fe3O4-LiMo3Se3 Nanoparticle Clusters as Superparamagnetic Nanocompasses. Langmuir. 2005;21:9709 - 13.

[87] Yokota K, Morvan M, Berret J-F, Oberdisse J. Stabilization and controlled association of inorganic nanoparticles using block copolymers. Europhys Lett. 2005;69:284-90.

[88] Sacanna S, Philipse AP. Preparation and Properties of Monodisperse Latex Spheres with Controlled Magnetic Moment for Field-Induced Colloidal Crystallization and (Dipolar) Chain Formation. Langmuir. 2006;22:10209 16.

[89] Sheparovych R, Sahoo Y, Motornov M, Wang SM, Luo H, Prasad PN, et al. Polyelectrolyte stabilized nanowires from $\mathrm{Fe} 3 \mathrm{O} 4$ nanoparticles via magnetic field induced self-assembly. Chemistry of Materials. 2006;18:591 - 3 .

[90] Berret J-F. Stoichiometry of electrostatic complexes determined by light scattering. Macromolecules. 2007;40:4260-6.

[91] Ge J, Hu Y, Yin Y. Highly Tunable Superparamagnetic Colloidal Photonic Crystals. Angewandte Chemie International Edition. 2007;46:7428 - 31.

[92] Corr SA, Byrne SJ, Tekoriute R, Meledandri CJ, Brougham DF, Lynch M, et al. Linear Assemblies of Magnetic Nanoparticles as MRI Contrast Agents. J Am Chem Soc. 2008;130:4214 - 5.

[93] Sen D, Spalla O, Belloni L, Charpentier T, Thill A. Temperature effects on the composition and microstructure of spray-dried nanocomposite powders. Langmuir. 2006;22:3798-806.

[94] Qi L, Chapel JP, Castaing JC, Fresnais J, Berret J-F. Stability and adsorption properties of electrostatic complexes: Design of hybrid nanostructures for coating applications. Langmuir. 2007;23:11996-8.

[95] Fresnais J, Berret J-F, Qi L, Chapel J-P, Castaing J-C, Sandre O, et al. Universal scattering behavior of coassembled nanoparticle-polymer clusters. Phys Rev E. 2008;78:040401.

[96] Qi L, Chapel JP, Castaing JC, Fresnais J, Berret J-F. Organic versus hybrid coacervate complexes: coassembly and adsorption properties. Soft Matter. 2008;4:577-85.

[97] Qi L, Sehgal A, Castaing JC, Chapel JP, Fresnais J, Berret JF, et al. Redispersible hybrid nanopowders: Cerium oxide nanoparticle complexes with phosphonated-PEG oligomers. Acs Nano. 2008;2:879-88.

[98] Fresnais J, Lavelle C, Berret JF. Nanoparticle Aggregation Controlled by Desalting Kinetics. The Journal of Physical Chemistry C. 2009;113:16371-9.

[99] Berret J-F, Sehgal A, Morvan M, Sandre O, Vacher A, Airiau M. Stable oxide nanoparticle clusters obtained by complexation. J Colloid Interface Sci. 2006;303:315-8. 
[100] Berret J-F, Sandre O, Mauger A. Size distribution of superparamagnetic particles determined by magnetic sedimentation. Langmuir. 2007;23:2993-9.

[101] Fresnais J, Berret J-F, Frka-Petesic B, Sandre O, Perzynski R. Electrostatic Co-Assembly of Iron Oxide Nanoparticles and Polymers: Towards the Generation of Highly Persistent Superparamagnetic Nanorods. Adv Mater. 2008;20:3877-81.

[102] Fresnais J, Berret J-F, Frka-Petesic B, Sandre O, Perzynski R. Reorientation kinetics of superparamagnetic nanostructured rods. Journal of Physics: Condensed Matter. 2008:494216.

[103] Chanteau B, Fresnais J, Berret JF. Electrosteric Enhanced Stability of Functional Sub-10 nm Cerium and Iron Oxide Particles in Cell Culture Medium. Langmuir. 2009;25:9064-70.

[104] Fresnais J, Ishow E, Sandre O, Berret J-F. Electrostatic Co-assembly of Magnetic Nanoparticles and Fluorescent Nanospheres: A Versatile Approach Towards Bimodal Nanorods. Small. 2009;5:2533-6.

[105] Berret J-F, Yokota K, Morvan M, Schweins R. Polymer-nanoparticle complexes: From dilute solution to solid state. J Phys Chem B. 2006;110:19140-6.

[106] Massart R. Préparation de Ferrofluides Aqueux en l'Absence de Surfactant: Comportement en Fonction du pH et de la Nature des Ions Présents en Solution. C R Acad Sci (Paris). 1980;291:1 - 3.

[107] Massart R, Dubois E, Cabuil V, Hasmonay E. Preparation and properties of monodisperse magnetic fluids. J Magn Magn Mat. 1995;149:1 - 5.

[108] Bee A, Massart R, Neveu S. Synthesis of very fine maghemite particles. J Magn Magn Mat. 1995;149:6 - 9.

[109] Dubois E, Cabuil V, Boue F, Perzynski R. Structural analogy between aqueous and oily magnetic fluids. J Chem Phys. 1999;111:7147 - 60.

[110] Lucas IT, Durand-Vidal S, Dubois E, Chevalet J, Turq P. Surface Charge Density of Maghemite Nanoparticles:, Äâ Role of Electrostatics in the Proton Exchange. The Journal of Physical Chemistry C. 2007;111:18568-76.

[111] Jacquin M, Muller P, Talingting-Pabalan R, Cottet H, Berret J-F, Futterer T, et al. Chemical analysis and aqueous solution properties of charged amphiphilic block copolymers PBA-b-PAA synthesized by MADIX((c)). J Colloid Interface Sci. 2007;316:897-911.

[112] Klein R, D’aguanno B. Static Scattering Properties of Colloidal Suspensions. In: Brown W, (editor). Light Scattering, Principles and Developments. Oxford1996. p. 30 - 102.

[113] Holde KEv. Chromatin. New York: Springer-Verlag; 1989.

[114] Burton DR, Butler MJ, Hyde JE, Phillips D, Skidmore CJ, Walker IO. Interaction of Core Histones with DNA - Equilibrium Binding Studies. Nucleic Acids Research. 1978;5:3643-63.

[115] Sehgal A, Lalatonne Y, Berret J-F, Morvan M. Precipitation-redispersion of cerium oxide nanoparticles with poly(acrylic acid): Toward stable dispersions. Langmuir. 2005;21:9359-64.

[116] Yan M, Fresnais J, Berret JF. Growth mechanism of nanostructured superparamagnetic rods obtained by electrostatic co-assembly. Soft Matter. 2010;6:1997-2005.

[117] Fung AO, Kapadia V, Pierstorff E, Ho D, Chen Y. Induction of Cell Death by Magnetic Actuation of Nickel Nanowires Internalized by Fibroblasts. The Journal of Physical Chemistry C. 2008;112:15085-8.

[118] Loose W, Ackerson BJ. Model-calculations for the analysis of scattering data from layered structures. J Chem Phys. 1994;101:7211-20.

[119] Molino FR, Berret JF, Porte G, Diat O, Lindner P. Identification of flow mechanisms for a soft crystal. Eur Phys J B. 1998;3:59-72.

[120] Johnson BK, Prud'homme RK. Mechanism for Rapid Self-Assembly of Block Copolymer Nanoparticles. Phys Rev Lett. 2003;91:118302.

[121] Destremaut F, Salmon JB, Qi L, Chapel JP. Microfluidics with on-line dynamic light scattering for size measurements. Lab Chip. 2009;9:3289-96. 